\title{
Collagenous scaffolds supplemented with hyaluronic acid and chondroitin sulfate used for wound fibroblast and embryonic nerve cell culture
}

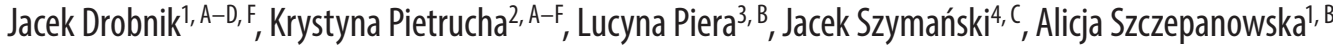 \\ ${ }^{1}$ Laboratory of Connective Tissue Metabolism, Department of Neuropeptides Research, Medical University of Lodz, Poland \\ 2 Department of Material and Commodity Sciences and Textile Metrology, Lodz University of Technology, Poland \\ ${ }^{3}$ Department of Neuropeptides Research, Medical University of Lodz, Poland \\ ${ }^{4}$ Central Scientific Laboratory, Medical University of Lodz, Poland
}

A - research concept and design; $B$ - collection and/or assembly of data; $C$ - data analysis and interpretation; $D$ - writing the article; $E$ - critical revision of the article; $F$ - final approval of article

\author{
Address for correspondence \\ Jacek Drobnik \\ E-mail: drob@op.pl \\ Funding sources \\ This work was supported by National Science Center Grant No. \\ DEC 2011/03/B/ST8/05867 \\ Conflict of interest \\ none declared

\section{Acknowledgements} \\ The authors would like to thank Henryk Wrzosek, MSc, for \\ providing scanning electron microscope facilities. The authors \\ are also grateful to Teresa Staszewska for her excellent technical \\ assistance.
}

Received on November 10, 2015

Revised on March 15, 2016

Accepted on April 25, 2016

\begin{abstract}
Background. Tissue engineering is a strategy aimed at improving the regeneration of injured tissues.

Objectives. The aim of the present study was to determine whether a tri-copolymer composed of crosslinked collagen, chondroitin sulfate and hyaluronic acid ( $\mathrm{COl}+\mathrm{CS}+\mathrm{HA}$ ) provides a better environment for fibroblast and embryonic nerve cell culture than a collagenous scaffold (Col).

Material and methods. The porosity of each of the matrices was characterized with a scanning electron microscope. Fibroblasts were isolated from rat wound granulation tissue (polypropylene net implanted subcutaneously). Embryonic nerve cells were obtained from the brains of rat embryos. The cells were applied to scaffolds and then stained with bisbenzimide to calculate cell entrapment within the material. The metabolic activity of the cells cultured within the scaffolds was tested using the 3-(4,5-dimethythiazol2-yl)-2,5-diphenyl tetrazolium bromide (MTT) assay.
\end{abstract}

Results. The Col scaffolds had a homogenously porous structure with a pore diameter of $50 \mu \mathrm{m}$ for $70 \%$ of pores. The pore diameter in the tri-copolymer (Col + HA + CS) ranged from 24 to $160 \mu \mathrm{m}(95 \%$ of total pore volume). Four times more cells (fibroblasts and embryonic nerve cells) were trapped within the superficial part of the collagenous scaffold than that of the tri-copolymer. On the third day of culture the metabolic activity of the fibroblasts within the 2 tested scaffolds was significantly higher than in the control conditions (cell culture on a laminin-coated surface). Also, the embryonic nerve cells demonstrated increased metabolic activity in Col + CS + HA scaffolds than the Col scaffolds.

Conclusions. Both fibroblasts and embryonic nerve cells could be seeded within the 2 tested scaffolds. Both the scaffolds provide good conditions for fibroblast culture. However, the Col + CS + HA tri-copolymer is preferable for embryonic nerve cell engineering.

Key words: collagen, fibroblasts in vitro, embryonic nerve cells

DOI

10.17219/acem/62835

Copyright

Copyright by Author(s)

This is an article distributed under the terms of the

Creative Commons Attribution Non-Commercial License

(http://creativecommons.org/licenses/by-nc-nd/4.0/) 
Large wounds lead to disturbances of homeostasis. Immediately following an injury, hemostasis and inflammation are induced. These processes are followed by cell migration and proliferation phases, after which fibroblasts begin the synthesis of an extracellular matrix. Together, these processes lead to scar formation that rearranges the continuity of the tissue. ${ }^{1}$ Large wounds heal very slowly due to the large space that needs to be covered by the scar. Although limited results can be obtained by treating large wounds with conventional methods, tissue engineering provides a chance to achieve better outcomes.

Cell engineering is a new strategy developed to regenerate nerve tissues. The engineered cells are supported by a biodegradable scaffold that not only forms a mechanical strut, but also may exert a regulatory influence on cell migration, proliferation and extracellular matrix synthesis. Thus, the implants obtained not only close the wound but also stimulate the self-repair or self-regeneration of the wound. ${ }^{2}$

The favorable physical and chemical properties of collagenous ( $\mathrm{Col}$ ) scaffolds for regenerative medicine have been described by Pietrucha et al. ${ }^{3-6}$ The Col materials create a supportive microenvironment for fibroblasts, osteoblasts and embryonic nerve cells. ${ }^{2,6-8}$ Wound fibroblasts are thought to enter the scaffold from the margins of the wound and participate in scar formation. Collagen may regulate the function of cells in the wound by binding to the integrin subfamily $(\alpha 1 \beta 2, \alpha 2 \beta 2, \alpha 10 \beta 2$ and $\alpha 11 \beta 2)$, which would influence granulation tissue remodeling, angiogenesis, fibroblast adhesion and myofibroblast differentiation in the wound. ${ }^{9}$

Hyaluronic acid (HA) is a polysaccharide composed of glucuronic acid and $\mathrm{N}$-acetylglucosamine. A scaffold containing hyaluronic acid promotes wound repair in rats, accelerates wound closure, supports re-epithelialization and angiogenesis in mice, and stimulates fibroblasts to release growth factors in rats. ${ }^{10-11}$ Chondroitin sulfate (CS), composed of glucuronic acid and $\mathrm{N}$-acetylgalactosamine, was found to promote fibrotic scar formation in an injury of the central nervous system. ${ }^{12} \mathrm{CS}$ is negatively charged due to the presence of $\mathrm{SO}_{4}{ }^{2-}$ and $\mathrm{COO}^{-}$moieties in the molecule, and this negative polarity allows the adhesion of growth factors and cells. In addition, CS influences the migration of cells. ${ }^{13}$

Some studies report that scaffolds composed of collagen, HA and CS have beneficial effects when used for skin or cartilage regeneration. ${ }^{14,15}$ The materials were seeded with skin fibroblasts or adipose-derived stem cells. ${ }^{14}$ Li et al. constructed a scaffold composed of collagen, hyaluronic acid and chondroitin-6-sulfate for nucleus pulposus tissue engineering. The copolymer was characterized by a highly porous structure, hydrophilic properties, good mechanical stability, low immunogenicity and good biocompatibility. ${ }^{16}$

The aim of the present study was to determine whether supplementation of a Col scaffold with CS and HA can in- fluence the biological properties necessary for fibroblast and embryonic nerve cell culture, so that the scaffold providing the best environment for a given purpose can be selected. A previous study found that a scaffold composed of collagen cross-linked with CS constitutes a good environment for embryonic nerve cells. ${ }^{6}$ A novel element of the present study was that it investigated whether a collagen tri-copolymer with the addition of $\mathrm{HA}$ and $\mathrm{CS}$ $(\mathrm{Col}+\mathrm{HA}+\mathrm{CS})$ can enhance cultures not only of embryonic nerve cells, but also wound fibroblasts. In this way, the study determined the usefulness of this tri-copolymer for tissue engineering.

\section{Material and methods}

\section{The animals}

The experiments were performed on 7 male and 9 female Wistar rats weighing $230 \pm 40 \mathrm{~g}$. The animals were kept on a 12 : 12 light/dark cycle (from 7:00) and were given free access to standard rat feed (Bakutil, Radzyń Podlaski, Poland) and tap water ad libitum. The study was approved by the Local Ethics Committee in Łódź, Poland.

\section{Wound model}

A $4 \mathrm{~cm}$ skin incision was made in the left lumbar region of each anesthetized rat and $3 \times 2 \mathrm{~cm}$ polypropylene mesh was inserted subcutaneously. The incision was closed with 4 silk sutures. Four weeks after the wound was made, the implant, overgrown by connective tissue, was removed under aseptic conditions. ${ }^{17}$

\section{Fibroblast isolation and culture}

The removed implants containing granulation tissue were stored in RPMI 1640 medium (Gibco, Paisley, UK) containing gentamicin $(25 \mu \mathrm{g} / \mathrm{mL})$ and fungizone $(2.5 \mu \mathrm{g} / \mathrm{mL})$. After mincing, the tissue was incubated in $0.1 \%$ collagenase solution $\left(37^{\circ} \mathrm{C}, 5 \% \mathrm{CO}_{2}\right)$ for $40 \mathrm{~min}$. After centrifugation (5 min, $1000 \mathrm{rpm}$ ), the samples pellets were washed with Dulbecco's modified Eagle's medium (DMEM) containing 10\% fetal calf serum, gentamicin and fungizone (Gibco, Paisley, UK), then centrifuged and plated on dishes. The non-adherent cells were washed out after $2 \mathrm{~h}$. The cells were cultured in a $\mathrm{CO}_{2}$ incubator at $37^{\circ} \mathrm{C}$ in a $100 \%$ humidified atmosphere of $5 \% \mathrm{CO}_{2}$ and 95\% air. Six-well plates (TPP Techno Plastic Products AG, Trasadingen, Switzerland) were used for cell culture.

After reaching confluence, the cells were trypsinized and passaged to new flasks. New cultures were set up at an initial cell density of $8 \times 10^{3} / \mathrm{cm}^{2}$. Bürker's chamber was used for cell counting. Necrotic cells were stained using trypan blue elimination assays. After the second passage, the experiments were carried out in DMEM contain- 
ing $3 \%$ calf serum and antibiotics at the concentrations given above; the calf serum concentration was lowered to decrease interference from fibroblast-scaffold interactions. The isolated cells were identified as fibroblasts as described in an earlier study by the current authors. ${ }^{17}$ Both fibroblasts and embryonic nerve cells were used in 2 experiments for cell entrapment and for 3-(4,5-dimethythiazol-2-yl)-2,5-diphenyl tetrazolium bromide (MTT) assays. Each experimental group consisted of 7 wells with the cells of different animals.

\section{Isolation and culture of embryonic nerve tissue}

Pregnant Wistar rats were euthanized and embryos at day 17 of gestation were isolated from the uterus in cold phosphate buffered saline (PBS, Gibco, Paisley, UK). After removal of the brains, the meninges and blood vessels were excised. The brain samples were incubated with $1 \mathrm{~mL}$ of enzymatic solution composed of collagenase $(1 \mathrm{mg} / \mathrm{mL})$, (Sigma, St. Louis, USA) and dispase $(2 \mathrm{mg} / \mathrm{mL})$, (Gibco, Paisley, UK) at room temperature $(5 \mathrm{~min})$ to remove the connective tissue. The brains were then incubated with $2 \mathrm{~mL}$ trypsin $\left(5 \mathrm{~min}\right.$ at $\left.37^{\circ} \mathrm{C}\right)$, (Gibco, Paisley, UK). The trypsin was neutralized with $5 \mathrm{~mL}$ of medium, (MACS NeuroBrew-21, Miltenyi Biotec, Bergisch Gladbach, Germany) after which the sample was centrifuged at $1000 \mathrm{rpm}$.

The cells were resuspended with $100 \mu \mathrm{L}$ of medium (MACS NeuroBrew-21, Miltenyi Biotec, Bergisch Gladbach, Germany) and gently triturated (3-5 X). The cells were then seeded on laminin-coated 6-well plates (TPP, Trasadingen, Switzerland).

The embryonic nerve cells were cultured in MACS NeuroBrew-21 containing gentamicin $(25 \mu \mathrm{g} / \mathrm{mL})$ and fungizone $(2.5 \mu \mathrm{g} / \mathrm{mL})$. The phenotype of the cells was determined by flow cytometry. The entrapment study and MTT experiments were performed on cultures of cells derived from 9 animals.

\section{Flow cytometry experiments}

Flow cytometry experiments were performed to determine the phenotype of the isolated embryonic nerve cells. After fixation with BD Cytofix (BD Biosciences, Franklin Lakes, USA), the embryonic nerve cells were permeabilized by incubation with BD Phosflow Perm Buffer III (BD Biosciences, Franklin Lakes, USA). The samples were then washed with PBS and centrifuged (5 min, $1000 \mathrm{rpm}$ ).

The permeabilized cells were stained $\left(4-8^{\circ} \mathrm{C}\right.$ for $\left.30 \mathrm{~min}\right)$ in BD Pharmingen stain buffer (BD Biosciences, Franklin Lakes, USA), with the following antibodies:

- mouse anti-Microtubule-Associated Protein 2B (MAP2B) conjugated with Alexa Fluor 488 (BD Biosciences, Franklin Lakes, USA);

- mouse anti-Myelin Basic Protein (MBP) conjugated with PE (Abcam, Cambridge, USA);
- mouse anti-Glial Fibrillary Acidic Protein (GFAP) conjugated with PE (BD Pharmingen, San Diego, USA);

- goat anti-mouse IgG2b heavy chain isotype control conjugated with FITC (BD Pharmingen, San Diego, USA);

- mouse IgG2b isotype control conjugated with PE (BD Pharmingen, San Diego, USA);

- mouse IgG1 k isotype control conjugated with Alexa Fluor 488 (BD Pharmingen, San Diego, USA).

The stained cells $\left(3 \times 10^{4} /\right.$ samples $)$ were analyzed using a Becton-Dickinson FACScan analytical flow cytometer (Becton Dickinson, Franklin Lakes, USA).

\section{Preparation and characterization of ternary composite collagen-based scaffolds}

The collagen/hyaluronic acid/chondroitin sulfate ( $\mathrm{Col}+$ $+\mathrm{HA}+\mathrm{CS}$ ) matrices were constructed as described previously, with some modifications. ${ }^{18}$ In brief, a dispersion in dilute acetic acid ( $\mathrm{pH}$ 3.2) of type I porcine collagen (Col) (Euroimplant, Rybie, Poland), hyaluronic acid (HA) from Streptococcus equi with molecular weight of $1.6 \times 10^{3} \mathrm{kDa}$, (Sigma-Aldrich, St. Louis, USA) and chondroitin sulfate (CS) from shark cartilage with an average molecular weight of $20 \mathrm{kDa}$, (Sigma-Aldrich, St. Louis, USA) at a ratio of $6: 1: 1$ was freeze-dried at $-50^{\circ} \mathrm{C}$ to form 3D sponge-shaped porous scaffolds. For comparison, sponges from pure Col were also prepared. To improve the functional properties of the scaffolds, all the collagenous sponges were treated with a carbodiimidebased process as previously described, but with significant modifications: The sponges were cross-linked at room temperature by immersion in an $80 \%$ ethanol-water solution containing $33 \mathrm{mM}$ 1-ethyl-3-(3-dimethyl aminopropyl) carbodiimide hydrochloride (EDC) and $6 \mathrm{mM}$ $\mathrm{N}$-hydroxysuccinimide (NHS). ${ }^{19,20}$ After the reaction, the matrices were thoroughly washed in $0.1 \mathrm{M} \mathrm{Na}_{2} \mathrm{HPO}_{4}$, and then with deionized water. Finally, the cross-linked matrices were again lyophilized at $-55^{\circ} \mathrm{C}$. All the collagenbased scaffolds were sterilized with ethylene oxide (EtO). The multi-component porous sponges were then applied as matrices for culturing cells to form a hybrid tissue-like structure intended for cell engineering.

As noted above, the addition of hyaluronic acid to an earlier investigated scaffold $(\mathrm{Col}+\mathrm{CS})$ created a tri-copolymer $(\mathrm{Col}+\mathrm{CS}+\mathrm{HA})$, the potential of which for embryonic nerve cell and wound fibroblast culture had not been investigated prior to the present study. ${ }^{6}$

The porosity of each of the matrices was characterized using a Nova NanoSEM 230 scanning electron microscope (FEI Company, Eindhoven, the Netherlands) with an Apollo 40 SDDEDS X-ray microanalyser (EDAX McKee Drive Mahwah, USA). The operating parameters were as follows: A low vacuum setting of $70 \mathrm{~Pa}$, an accelerating voltage of $10 \mathrm{kV}$ and LVD detector selected. Crosssections or surface sections of the sponge were obtained 
by breaking the specimens after freezing them in liquid nitrogen. An image analysis application was used to determine the average diameter of the pores, with at least 200 pores assessed on each specimen at $\times 100$ and $\times 400$ magnification. Fig. 1 shows the results of the pore analysis of 2 collagen-based scaffolds.

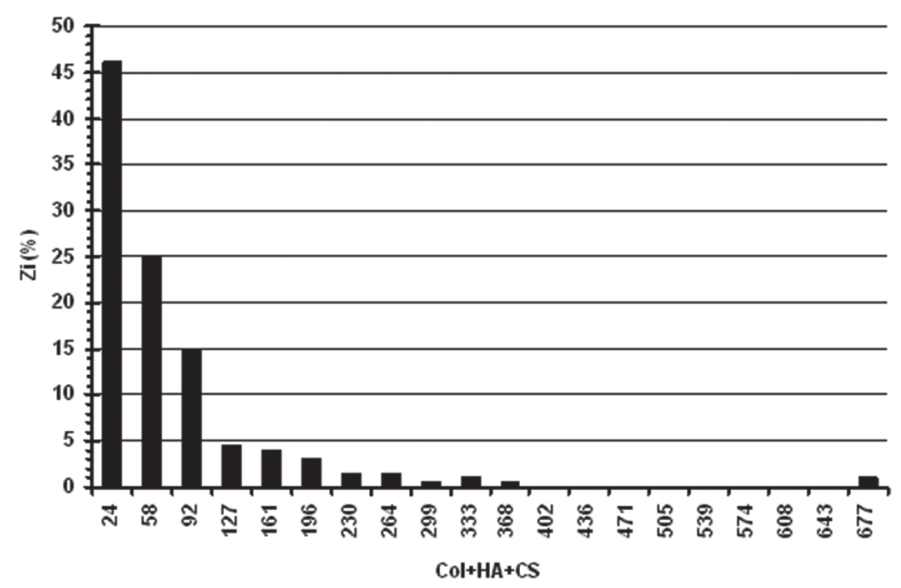

Fig. 1. Distribution of pore diameters of collagen-based scaffolds modified with EDC and NHS: (K1) scaffold composed of collagen

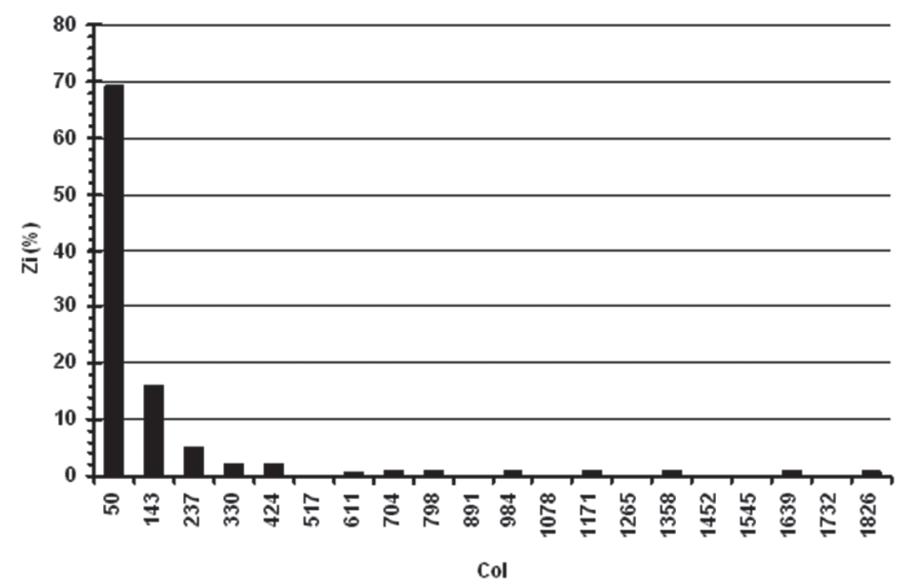

Fig. 2. Distribution of pore diameters of collagen-based scaffolds modified with EDC and NHS: (K2) tri-copolymer scaffold composed of collagen, chondroitin sulfate and hyaluronic acid

\section{Cell entrapment study}

Wound fibroblasts were seeded on the scaffolds at a density of $10^{4} /$ well of 96-well plates (TPP, Trasadingen, Switzerland) and incubated for $24 \mathrm{~h}$ in DMEM containing $3 \%$ fetal calf serum, gentamicin and fungizone $\left(37^{\circ} \mathrm{C}\right.$, $5 \% \mathrm{CO}_{2}$ ). Embryonic nerve cells were added to a 96-well plate at a density of $10^{4}$ cells per well. They were then incubated for $24 \mathrm{~h}$ in MACS NeuroBrew-21 medium (Miltenyi Biotec, Bergisch Gladbach, Germany). After being washed with PBS, the samples were incubated for $30 \mathrm{~min}$ $\left(37^{\circ} \mathrm{C}, 5 \% \mathrm{CO}_{2}\right)$ with bisbenzimide $(1 \mu \mathrm{g} / \mathrm{mL}$ in PBS). After incubation, the scaffolds were washed with PBS, and the stained nuclei of the fibroblasts were counted under the microscope at $\times 100$ magnification. ${ }^{3}$

\section{MTT experiments}

The experiments were performed in 96-well plates (TPP, Trasadingen, Switzerland). The cells were applied to the scaffold at a density of $10^{4}$ cells per well. After 2 or 3 days of culture, the samples were washed 3 times with PBS, and then $50 \mu \mathrm{L}$ of 3-(4,5-dimethythiazol-2-yl)-2,5diphenyl tetrazolium bromide (MTT reagent, $5 \mathrm{mg} / \mathrm{mL}$, Sigma, St. Louis, USA) was added to each well. The samples were then incubated for $4 \mathrm{~h}$ at $37^{\circ} \mathrm{C}$ in a humid atmosphere containing 5\% $\mathrm{CO}_{2}$. The MTT solution was removed and $200 \mu \mathrm{L}$ DMSO was added to each sample and incubated for $10 \mathrm{~min}$. After the addition of Sorensen's buffer $\left(0.1 \mathrm{M} \mathrm{NaCl}+0.1 \mathrm{M} \mathrm{C}_{2} \mathrm{H}_{5} \mathrm{NO}_{2} ; \mathrm{pH}=10.5\right)$, the absorbance was determined at a wavelength of $570 \mathrm{~nm}$ using an EL $\times$ 800UV Universal Microplate Reader (BioTek Instruments Inc., Winooski, USA). Three groups of cells were studied: control cultures (2D), cultures on Col scaffolds and cultures on Col + CS + HA scaffolds. The control cells were seeded on a laminin-coated surface.

\section{Statistical analysis}

The Kruskal-Wallis test was used for the statistical analysis. Statistical differences between more than 2 independent groups were evaluated by multiple comparisons of mean ranks. The U-Mann Whitney test was used for the evaluation of statistical differences between 2 independent groups. The statistical differences between 2 dependent groups were calculated using the t-test for 2 dependent samples (when the data distribution was normal) or the Wilcoxon test (when the data distribution was not normal). The threshold of significance was $p<0.05$. The calculations were performed using STATISTICA 12.5 software (Dell Statistica, Tulsa, USA).

\section{Results}

The Col scaffolds were found to have a homogenously porous structure with a pore diameter of $50 \mu \mathrm{m}$ in $70 \%$ of the pores. For $15 \%$ of the total pore volume, the pore diameter ranged from $50 \mu \mathrm{m}$ to $140 \mu \mathrm{m}$. However, for the tri-copolymer $(\mathrm{Col}+\mathrm{HA}+\mathrm{CS})$, the pore diameter ranged from 24 to $160 \mu \mathrm{m}$ for $95 \%$ of the total pore volume of the scaffold (Figs. 1 and 2).

Fibroblasts were isolated from the granulation tissue of the wound. The phenotype of the cells was confirmed by electron microscopy as described in an earlier study. ${ }^{17}$ The embryonic nerve cells were differentiated into neurons (MAP2B positive cells, Fig. 3), oligodendrocytes (MBP positive cells, Fig. 4) and astrocytes (GFAP positive cells, data not shown). ${ }^{6}$

Fibroblasts were able to enter both $\mathrm{Col}$ and $\mathrm{Col}+\mathrm{CS}+$ + HA scaffolds, but the Col + CS + HA scaffolds were less populated on the upper surface. Statistically signifi- 

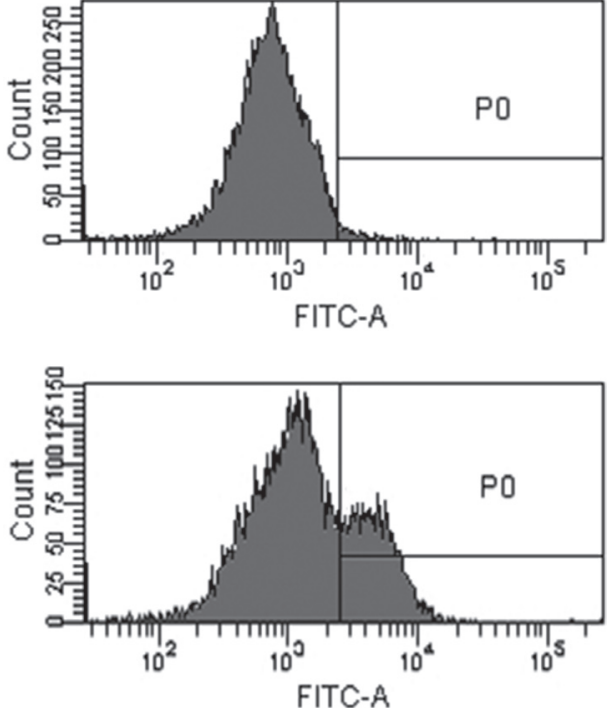

Fig. 3. Fluorescence-activated cell sorting (FACS) profiles showing the expression of microtubule-associated protein (MAP2b) on embryonic nerve cells (lower panel) compared with the control (upper panel)
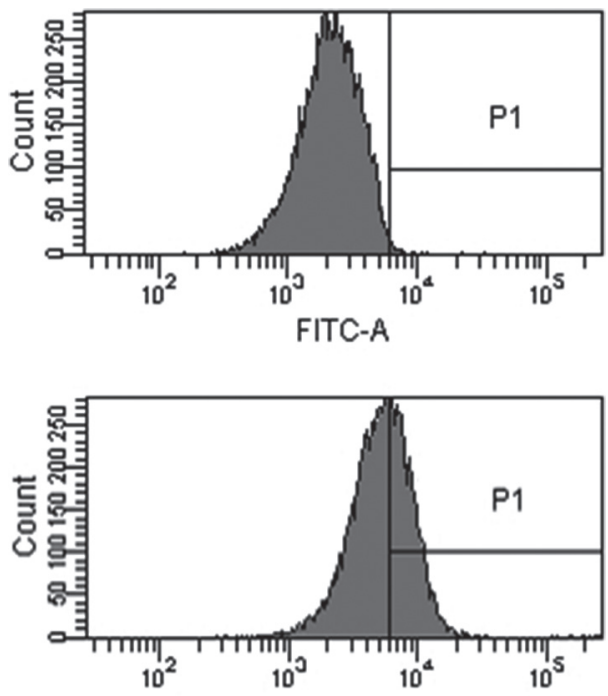

FITC-A

Fig. 4. FACS profiles showing the expression of myelin basic protein (MBP) on embryonic nerve cells (lower panel) compared with the control (upper panel)

cant differences were observed in the numbers of fibroblasts infiltrating the $\mathrm{Col}$ and $\mathrm{Col}+\mathrm{CS}+\mathrm{HA}$ scaffolds (Fig. 5). At the same time, the embryonic nerve cells were entrapped by both the Col and Col + CS + HA scaffolds, but significantly higher numbers of cells were found in the former than the latter (Fig. 6).

The metabolic activity of the cells was analyzed after 2 or 3 days of culture. On the $2^{\text {nd }}$ day, lower fibroblast metabolic activity was found in the $\mathrm{Col}+\mathrm{CS}+\mathrm{HA}$ scaffolds than in the controls (a 2D cell culture on a laminin-coated surface). On the $3^{\text {rd }}$ day, however, the metabolic activity of the fibroblasts in Col and Col + CS + HA scaffolds was higher than in the controls. Interestingly, while the meta-

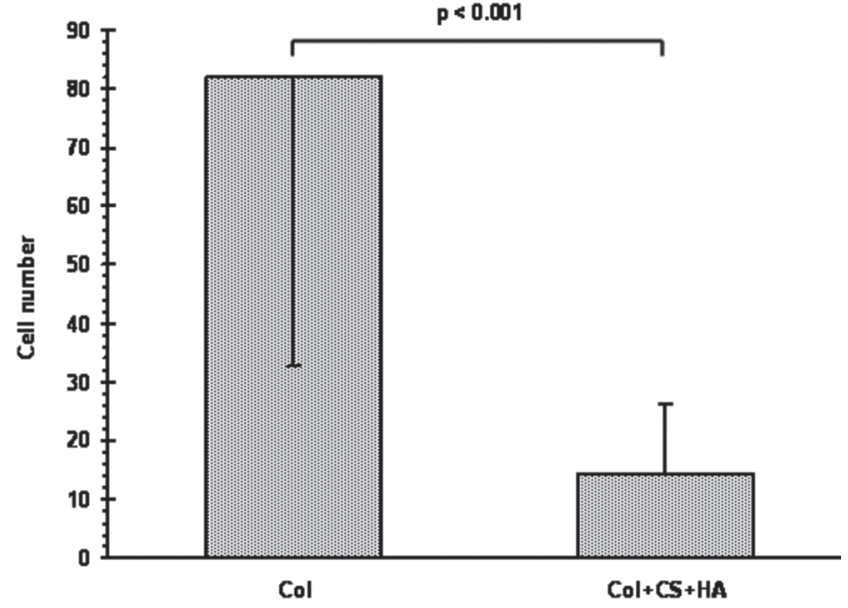

Fig. 5. Number of fibroblasts stained with bisbenzimide entrapped by scaffolds made of collagen $(\mathrm{Col})$ and of collagen with chondroitin sulfate and hyaluronic acid (Col + CS + HA). Each value expresses the mean of 6 samples \pm standard deviation (SD)

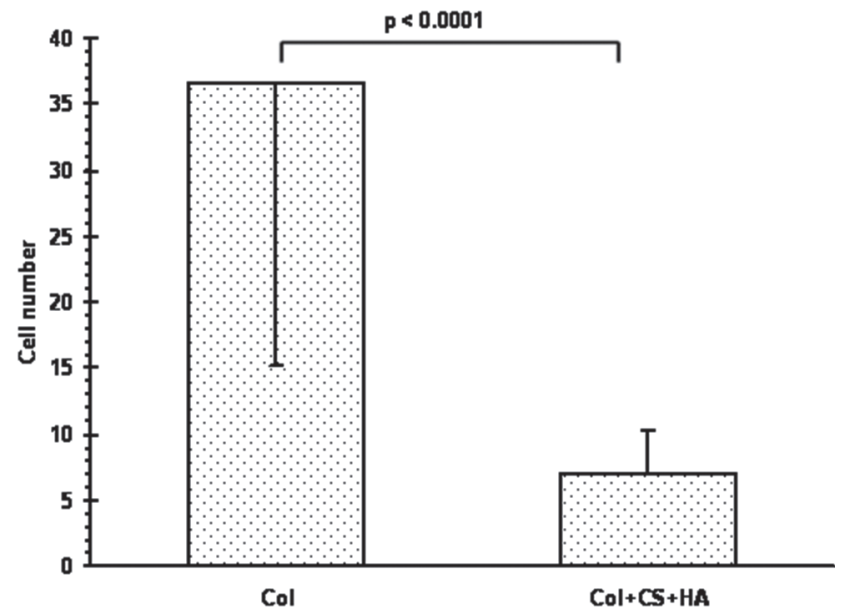

Fig. 6. Number of embryonic nerve cells stained with bisbenzimide entrapped by scaffolds made of collagen (Col) and of collagen with chondroitin sulfate and hyaluronic acid ( $\mathrm{Col}+\mathrm{CS}+\mathrm{HA})$. Each value expresses the mean of 6 samples \pm standard deviation (SD)

bolic activity of the fibroblasts fell from day 2 to day 3 in the control cultures, the opposite occurred in the scaffolds composed of Col + CS + HA, with fibroblast activity doubling from day 2 to day 3 (Fig. 7).

On the $2^{\text {nd }}$ and $3^{\text {rd }}$ days of culture, embryonic nerve cells within the Col scaffolds demonstrated decreased metabolic activity compared with the controls. In the Col scaffolds, the metabolic activity of the embryonic nerve cells was significantly higher on the $3^{\text {rd }}$ day of culture than on the $2^{\text {nd }}$ day. Within the Col $+\mathrm{CS}+\mathrm{HA}$ scaffolds, the embryonic nerve cells demonstrated increased metabolic activity on both days 2 and 3 of culture compared to the Col scaffolds (Fig. 8). Lower metabolic activity of both fibroblasts (Fig. 7) and embryonic nerve cells (Fig. 8) was noted on day 3 of culture, compared with day 2 in the control groups. 


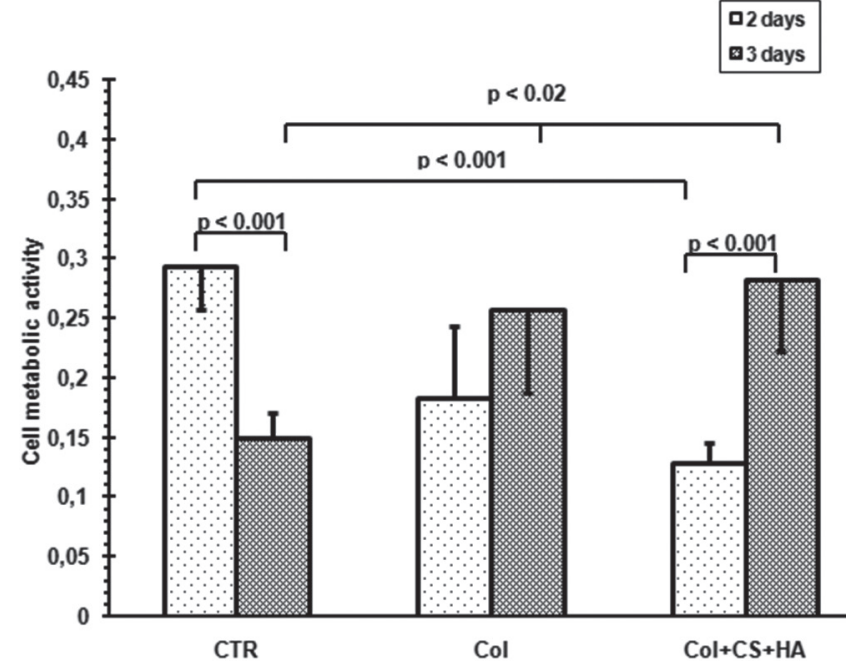

Fig. 7. Metabolic activity of fibroblasts cultured for 2 or 3 days on a laminincoated surface (CTR), in collagen scaffolds (Col) and in scaffolds composed of collagen, chondroitin sulfate and hyaluronic acid (Col + CS + HA). Each value expresses the mean of 8 samples \pm standard deviation (SD)

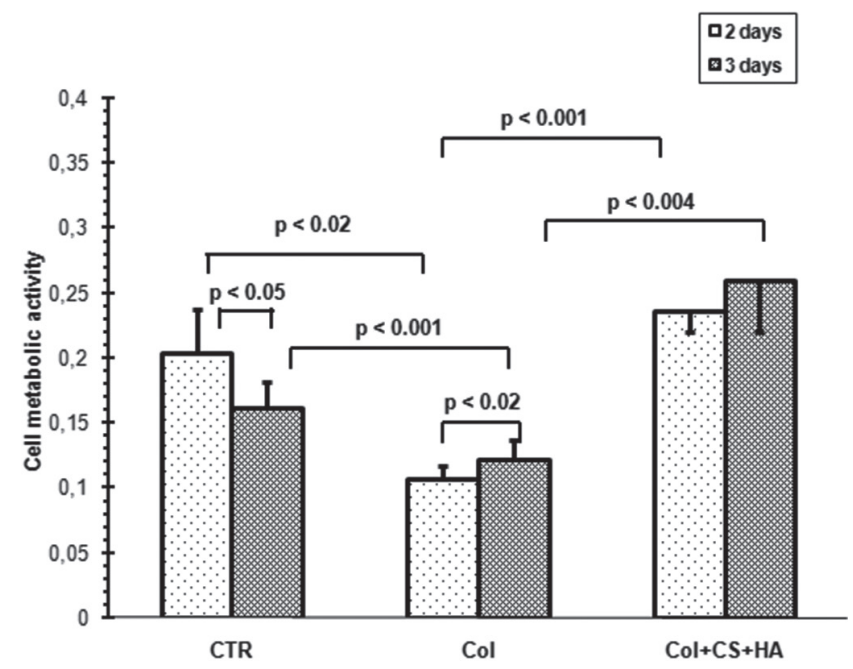

Fig. 8. Metabolic activity of embryonic nerve cells cultured for 2 or 3 days on a laminin coated surface (CTR), in collagen scaffolds (Col) and in scaffolds composed of collagen chondroitin sulfate and hyaluronic acid $(\mathrm{Col}+\mathrm{CS}+\mathrm{HA})$. Each value expresses the mean of 8 samples \pm standard deviation (SD)

\section{Discussion}

Earlier studies indicated that the secondary triple helix structure of collagen present within Col scaffolds remained unchanged, thus allowing the optimal biological properties of the scaffold to be used in regenerative medicine. ${ }^{3-6}$ In the present study, the presence or absence of cross-links, $\mathrm{CS}$ and HA in the collagen sponges had a significant effect on the overall structure and distribution of the interconnected pores (Figs. 1, 2). After cross-linking collagen alone, the sponges exhibited an almost homogenous distribution of pores, with $70 \%$ of the pores having a diameter of $50 \mu \mathrm{m}$ (Fig. 1, K1). Moreover, the results also showed that pore sizes ranging from 50 to $140 \mu \mathrm{m}$ made up about $15 \%$ of the total pore volume. Conversely, the pore size of the ternary composite Col-HA-CS sponges, cross-linked with EDC/ NHS, was significantly lower than in the collagen sponges without HA and CS (Fig. 2, K2). The largest fraction, comprising at least $70 \%$ of the total pore volume of the sponge, was occupied by pores ranging from 24 to $90 \mu \mathrm{m}$ in size. Only $15 \%$ of the Col + HA + CS scaffolds contained pores much larger than $90 \mu \mathrm{m}$, i.e. ranging between 127 and $196 \mu \mathrm{m}$. Wang et al. reported that the pores of tri-copolymer Col + CS + HA (9:1:1) scaffolds were uniform and widely interconnected, with a mean diameter of $109 \mu \mathrm{m} .{ }^{21}$ The dimensions of pores in collagenous scaffolds prepared by Yannas et al. ranged from 20 to $125 \mu \mathrm{m} .{ }^{22}$ Huang et al. observed that in a collagen and glycosaminoglycan copolymer, the pore size ranged from 20 to $140 \mu \mathrm{m} .^{23}$

The present study showed good fibroblast and embryonic nerve cell entrapment within the Col scaffolds (Figs. 5, 6). The presence of a relatively high density of cells settled within the Col material implies appropriate porosity, allowing the passage of different cell types into the scaffold. Moreover, more fibroblasts than embryonic nerve cells were entrapped in the Col scaffold. This effect is believed to be caused by the size and shape of fibroblasts, which may be a better fit for the pores. In addition, the $\mathrm{Col}+\mathrm{CS}+\mathrm{HA}$ scaffolds were about 4 times less populated than the Col scaffolds and controls. Thus, supplementation of the scaffold with chondroitin sulfate and hyaluronic acid decreases the ability of the Col scaffold to catch both fibroblasts and embryonic nerve cells. In a previous study, the numbers of embryonic nerve cells in the Col scaffolds and in a Col-chondroitin sulfate composite were found to be the same. ${ }^{6}$ In the present study, the addition of HA may disrupt the closed, honeycomb-like structure of the Col scaffold and cause the formation of open interconnections between pores, leading to the low populations of seeded fibroblasts and embryonic nerve cells observed in the tri-copolymer $\mathrm{Col}+\mathrm{CS}+\mathrm{HA}$ scaffolds. ${ }^{24}$ Although all the applied cells were entrapped within the scaffolds, different numbers of cells were found on the superficial parts of the scaffolds.

The metabolic activity of fibroblasts and embryonic nerve cells in the control groups (Figs. 6, 7) was found to be lower on the $3^{\text {rd }}$ day of culture than on the $2^{\text {nd }}$ day. This effect was probably caused by the limitations of a 2D culture and intensive cell-cell interactions. Bracke et al. reported inhibition of several energy-requiring activities in confluent epithelial cells, but MTT conversion increased only in confluent cells with a dysfunctional E-cadherin/ /catenin complex, implying that cell metabolism may be regulated by cell-cell adhesion via the E cadherin/catenin complex. ${ }^{25}$ Interestingly, this effect was not observed in 3D cultures created within the scaffolds (Figs. 7, 8). Thus, both $\mathrm{Col}$ and $\mathrm{Col}+\mathrm{CS}+\mathrm{HA}$ scaffolds provide favorable environments for fibroblast culture.

The results of the present study confirm that the metabolic activity of fibroblasts within the 2 types of scaffold were better on the $3^{\text {rd }}$ day than the $2^{\text {nd }}$ day of the culture 
(Fig. 7). In addition, on the $2^{\text {nd }}$ day of culture, the fibroblasts within the Col + CS + HA scaffolds demonstrated lower metabolic activity than in the controls. However, a rapid increase in metabolic activity was seen in the $\mathrm{Col}+\mathrm{CS}+\mathrm{HA}$ scaffolds on the $3^{\text {rd }}$ day of culture. These findings confirm those of earlier studies showing that a collagen-containing scaffold has good properties for tissue engineering. ${ }^{2,6,7}$ Fibroblasts seeded in collagen-gelatin sponges have been used as a drug delivery system, as they allow up-regulation of growth factor synthesis; these sponges appeared to be biocompatible with fibroblasts. ${ }^{26}$ Moreover, fibroblasts cultured within scaffolds with unidirectional collagen fibers secrete regulatory factors that modulate the wound-healing process. ${ }^{27}$ The collagen present in the extracellular space may also influence endogenous collagen expression. ${ }^{28}$

In the present study, the Col scaffolds did not guarantee good conditions for the culture of embryonic nerve cells: The cells seeded within the Col material displayed lower metabolic activity than in either the control cultures or the $\mathrm{Col}+\mathrm{CS}+\mathrm{HA}$ scaffolds. These results correspond with the data from the authors' previous study showing that embryonic nerve cells cultured within a scaffold composed of collagen and chondroitin sulfate demonstrate better metabolic activity than those in a Col scaffold. ${ }^{6}$ The authors therefore recommend the tri-copolymer for embryonic nerve cell engineering.

The authors' earlier study showed that a Col scaffold supplemented with CS provides good conditions for embryonic nerve cell culture. ${ }^{6}$ The addition of CS improved the metabolic activity of the cells compared with those in the Col scaffold. ${ }^{6}$ The present study found that cells seeded in tri-copolymer scaffolds demonstrated better metabolic activity than those in scaffolds composed of Col alone. This reaction is believed to be due to hyaluronic acid modifying the structure of collagen, allowing the formation of interconnections between pores, which supports the migration of cells to internal pores, allows for better communication between cells and improves the nutrition of the seeded cells. ${ }^{24}$ Since high concentrations of hyaluronic acid, chondroitin sulfate and heparin sulfate are known to be present in the brain, the collagenous scaffold was supplemented with glycosaminoglycans for nerve cell culture. ${ }^{29}$ Chondroitin sulfate is known to exert a regulatory influence on the differentiation and migration of endogenous neural precursor cells. ${ }^{23}$ In addition, stem cells seeded in scaffolds containing hyaluronic acid were characterized with better survival and differentiation. ${ }^{30}$

\section{Conclusions}

The study showed that fibroblasts and embryonic nerve cells were entrapped by both of the scaffolds used (Col and $\mathrm{Col}+\mathrm{CS}+\mathrm{HA})$. However, cell entrapment was about 4 times more efficient within the Col scaffolds than the
Col + CS + HA scaffolds for both of the tested cell cultures. Both the $\mathrm{Col}$ and $\mathrm{Col}+\mathrm{CS}+\mathrm{HA}$ scaffolds provided a good environment for fibroblasts; but since the scaffolds composed of $\mathrm{Col}+\mathrm{CS}+\mathrm{HA}$ guaranteed better conditions for the culture of embryonic nerve cells than Col alone, the tri-copolymer $\mathrm{Col}+\mathrm{CS}+\mathrm{HA}$ is recommended for the engineering of embryonic nerve cells.

\section{References}

1. Drobnik J. Wound healing and the effect of pineal gland and melatonin. J Exp Integr Med. 2012;2:3-14.

2. Guo Y, Zeng QCh, Zhang ChQ, et al. Extracellular matrix of mechanically streched cardiac fibroblasts improves viability and metabolic activity of ventricular cells. Int J Med Sci. 2013;10:1837-1845.

3. Pietrucha K. Physicochemical properties of 3D collagen-CS scaffolds for potential use in neural tissue engineering. Int J Biol Macrom. 2015;80:732-739.

4. Pietrucha K, Safandowska M. Dialdehyde cellulose-crosslinked collagen and its physicochemical properties. Process Biochem. 2015;50:2105-2111.

5. Pietrucha K. Changes in denaturation and rheological properties of collagen-hyaluronic acid scaffolds as a result of temperature dependencies. Int J Biol Macrom. 2005;36:299-304.

6. Pietrucha K, Szymański J, Drobnik J. The behavior of embryonic neural cells within the 3D micro-structured collagen-based scaffolds. International Federation for Medical and Biological Engineering (IFMBE) Proceedings. 2015;45:549-552.

7. Masci VL, Taddei AR, Gambellini G, Giorgi F, Fausto AM. Ultrastructural investigation on fibroblasts interaction with collagen scaffold. J Biomed Mater Res Part A. [published online September 16, 2015], doi: 10.1002/jbm.a.35563.

8. Quinlan E, Thompson EM, Matsiko A, O'Brien FJ, López-Noriega A. Functionalization of a collagen-hydroxyapatite scaffold with osteostatin to facilitate enhanced bone regeneration. Adv Healthc Mater. [published online September 28, 2015]. doi: 10.1002/adhm.201500439.

9. Koivisto L, Heino J, Hakkinen L, Larjava H. Integrins in wound healing. Adv Wound Care (New Rochelle). 2014;3(12):762-783.

10. Cerqueira MT, da Silva LP, Santos TC, et al. Human skin cell fractions fail to self-organize within a gellan gum/hyaluronic acid matrix but positively influence early wound healing. Tissue Eng Part A. 2014;20:1369-1378.

11. Kuroyanagi M, Yamamoto A, Shimizu N, et al. Development of antiadhesive spongy sheet composed of hyaluronic acid and collagen containing epidermal growth factor. J Biomater Sci Polym Ed. 2014;25:1253-1265.

12. Li HP, Homma A, Sango K, Kawamura K, Raisman G, Kawano H. Regeneration of nigrostriatal dopaminergic axons by degradation of chondroitin sulfate is accompanied by eliminationof the fibrotic scar and glia limitans in the lesion site. J Neurosci Res. 2007;85:536-547.

13. Zou XH, Jiang YZ, Zhang GR, Jin HM, Nguyen TM, Ouyang HW. Specific interactions between human fibroblasts and particular chondroitin sulfate molecules for wound healing. Acta Biomater. 2009;5: 1588-1595.

14. Dinescu S, Gălăţeanu B, Albu M, et al. Biocompatibility assessment of novel collagen-sericin scaffolds improved with hyaluronic acid and chondroitin sulfate for cartilage regeneration. Biomed Res Int. [published online November 7, 2013]. doi: 10.1155/2013/598056.

15. Murphy CM, Matsiko A, Haugh MG, Gleeson JP, O'Brien FJ. Mesenchymal stem cells fate is regulated by composition and mechanical properties of collagen-glycosaminoglycans scaffold. J Mech Behav Biomed Mater. 2012;11:53-62.

16. Li CQ, Huang B, Luo G, Zhang CZ, Zhuang Y ZO, Hou Y. Construction of collagen II/hyaluronate/chondroitin-6-sulfate tri-copolymer scaffold for nucleus pulposus tissue engineering and preliminary analysis of its physic-chemical properties and biocompatibility. J Mater Sci Mater Med. 2010;21:741-751.

17. Drobnik J, Owczarek K, Piera L, et al. Melatonin-induced augmentation of collagen deposition in cultures of fibroblasts and myofibroblasts is blocked by luzindole - melatonin membrane receptors inhibitor. Pharmacol Rep. 2013;65(3):642-649. 
18. Pietrucha K. Building up and characterization of multi-component collagen based scaffolds. International Federation for Medical and Biological Engineering (IFMBE) Proceedings. 2014;41:1626-1629.

19. Pieper JS, Oosterhof A, Dijkstra PJ, Veerkamp JH, van Kuppevelt $\mathrm{TH}$. Preparation and characterization of porous collagenous matrices containing bioavailable chondroitin sulfate. Biomaterials. 1999;20:847-858.

20. Harley BA, Leung JH, Silva ECCM, Gibson LJ. Mechanical characterization of collagen-glycosaminoglycan scaffolds. Acta Biomater. 2007;3:463-474.

21. Wang W, Zhang M, Lu W, et al. Cross-linked collagen-chondroitin sulphate-hyaluronic acid imitating extracellular matrix as scaffold for dermal tissue engineering. Tissue Eng Part C. 2010;16:269-279.

22. Yannas JV. Emerging rules for inducing organ regeneration. Biomaterials. 2013;34: 321-330.

23. Huang K.F, Hsu WCH, Chiu WT, Wang JY. Functional improvement and neurogenesis after collagen-GAG matrix implantation into surgical brain trauma. Biomaterials. 2012;33:2067-2075.

24. Tang S, Vickers SM, Hsu HP, Spector M. Fabrication and characterization of porous hyaluronic acid-collagen composite scaffolds. J Biomed Mater Res. 2007;82A:323-335.
25. Bracke $M E$, Depypere $H$, Labit $C$, et al. Functional downregulation of the E-cadherin/catenin complex leads to loss of contact inhibition of motility and of mitochondrial activity, but not of growth in confluent epithelial cell cultures. Eur J Cell Biol. 1997;74:342-349.

26. Hiwatashi N, Hirano S, Mizuta M, et al. Biocompatibility and efficacy of collagen/gelatin sponge scaffold with sustained release of basic fibroblast growth factor on vocal fold fibroblasts in 3-dimensional culture. Ann Otol Rhinol Laryngol. 2015;124(2):116-125.

27. Bustos RH, Suesca E, Millán D, González JM, Fontanilla MR. Realtime quantification of proteins secreted by artificial connective tissue made from uni- or multidirectional collagen I scaffolds and oral mucosa fibroblasts. Anal Chem. 2014;86(5):2421-2428.

28. Kuzan A, Smulczyńska-Demel A, Chwiłkowska A, Saczko J, Frydrychowski A, Dominiak M. An estimation of the biological properties of fish collagen in an experimental in vitro study. Adv Clin Exp Med. 2015;24:385-392.

29. Rauch U. Extracellular matrix components associated with remodeling processes in brain. Cell Mol Life Sci. 2004;61:2031-2045.

30. Ballios BG, Cooke MJ, van der Kooy D, Shoichet MS. A hydrogelbased stem cell delivery system to treat retinal degenerative diseases. Biomaterials. 2010;31:2555-2564. 